\title{
The impact of BMI on morbidity and mortality after femoral fractures
}

\author{
Sara S. Soliman ${ }^{1} \cdot$ Garrett B. Jordan ${ }^{1}$. Jaroslaw W. Bilaniuk ${ }^{1} \cdot$ Amanda Benfante $^{1} \cdot$ Karen Kong $^{1}$. \\ Rolando H. Rolandelli ${ }^{1}$. Terrence Curran ${ }^{1}$ - Zoltan H. Nemeth ${ }^{1}$ (I)
}

Received: 28 April 2021 / Accepted: 16 September 2021 / Published online: 12 October 2021

(c) Springer-Verlag GmbH Germany, part of Springer Nature 2021

\begin{abstract}
Purpose Femur fractures are the result of high energy injury and are associated with life-threatening complications. Therefore, we studied how body mass index (BMI) contributes to complications after femoral fractures.

Methods Using the 2016 American College of Surgeons Trauma Quality Improvement Program (ACS TQIP) database, we stratified 41,362 patients into groups based on their BMI: Normal Weight (NW), Overweight (OW), Obese (OB), Severely Obese (SO), and Morbidly Obese (MO). We compared each BMI group to the NW cohort for differences in demographic factors, comorbidities, complications, and mechanism of injury.

Results $\mathrm{OB}, \mathrm{SO}$, and MO patients sustained higher rates of traumatic injury from high energy mechanisms, such as motor vehicle trauma, in comparison to NW patients, who sustained more injuries from falls $(p<0.05)$. Correspondingly, obese patients were more likely than NW patients to sustain shaft and distal end fractures $(p<0.05)$. At hospital admission, obese patients presented with more comorbidities, such as bleeding disorders, congestive heart failure, diabetes mellitus, and hypertension $(p<0.05)$. Despite these individual findings, patients with OB, SO, and MO BMI, as opposed to NW BMI, were independently associated with a higher probability of developing at least one post-trauma complication. More specifically, MO patients were associated with a $45 \%$ higher odds of developing a complication $(p<0.05)$.

Conclusion Irrespective of presenting with more comorbidities and sustaining high energy injuries, OB, SO, and MO patients were independently associated with having a higher risk of developing complications following a femoral fracture. Overall, better clinical outcomes are observed among patients with no underlying conditions and normal BMI.
\end{abstract}

Keywords Femur fracture $\cdot$ Obesity $\cdot$ Complications $\cdot$ Comorbidity $\cdot$ Injury severity score

\section{Introduction}

The femur is the strongest and heaviest tubular bone, which is primarily responsible for weight-bearing and skeletal stability [1]. Consequently, femur fractures may cause tremendous impairment and can lead to substantial complications. Fractures of this nature have been increasingly prevalent in developed nations, with an annual incidence of 4.5 distal femur fractures per 100,000 and 129.4 proximal femur fractures per 100,000 [2].

Since the incidence of femoral fractures is increasing, it has become critical to elucidate factors that may affect the risk of sustaining these fractures and developing adverse

Zoltan H. Nemeth

zoltan.nemeth@atlantichealth.org

1 Department of Surgery, Morristown Medical Center, 100

Madison Ave. \#88, Morristown, NJ 07960, USA clinical outcomes. Previous publications have reported that conditions, such as osteoporosis [3], hypertension [4], diabetes [5], and several types of cancer [6], may heighten the risk of sustaining fractures. Unhealthy body mass index (BMI) (too high or too low) has also been notably cited as a risk factor for significant morbidity after sustaining a blunt traumatic injury $[7,8]$. In contrast, medical literature has also reported that obese patients, in comparison to normalweight patients, were associated with a lower risk of sustaining a hip fracture. It has been speculated that patients with obese BMIs are protected from these fractures due to their soft-tissue padding and high bone mineral density [9]. Understanding the exact role BMI plays in sustaining a femur fracture and post-trauma morbidity and mortality is critical as obesity has become a growing epidemic within the United States. For example, it has been recently reported that $63 \%$ of Americans are overweight, and $26 \%$ are obese [10]. Since the role of BMI in traumais unclear and the incidence 
of femur fractures is increasing, we sought to analyze the association between unhealthy BMI and the incidence of femur fractures and post-trauma morbidity and mortality using the 2016 American College of Surgeons Trauma Quality Improvement Program (ACS TQIP) database.

\section{Methods}

After obtaining approval from our Institutional Review Board, we collated all patients who sustained a femur fracture from the 2016 American College of Surgeons Trauma Quality Improvement Program (ACS TQIP). Patients ages 16 and older were then stratified into six groups according to their body mass index (BMI): Underweight (UW, BMI 10.00-18.49 kg/m²), Normal Weight (NW, BMI $18.50-24.99 \mathrm{~kg} / \mathrm{m}^{2}$ ), Overweight (OW, BMI $25.00-29.99 \mathrm{~kg} /$ $\mathrm{m}^{2}$ ), Obese (OB, BMI $30.00-34.99 \mathrm{~kg} / \mathrm{m}^{2}$ ), Severely Obese (SO, BMI $35.00-39.99 \mathrm{~kg} / \mathrm{m}^{2}$ ), and Morbidly Obese (MO, BMI $40.0-90.00 \mathrm{~kg} / \mathrm{m}^{2}$ ). Patients who sustained a femoral head/neck, trochanteric, shaft, and distal fracture were identified by abbreviated injury score (AIS) code classifications and included in our study. Patients who sustained multiple femur fractures or had fractures of an unspecified location were excluded.

Altogether, we compared each BMI cohort to the NW BMI cohort for differences in demographic factors, mechanism of injury (MOI), rates of comorbidities, and posttrauma complications. MOIs that were analyzed were falls, firearm, motor vehicle trauma (MVT) motorcyclist, MVT occupant, MVT other/unspecified, MVT pedal cyclist, MVT pedestrian, overexertion, pedal cyclist other, and pedestrian. Using Minitab 17, univariate analysis for categorical variables was completed with Chi-square test and Fischer's exact test, and all continuous variables were analyzed with Student's $t$-test. Multivariate logistic regression models comparing each BMI cohort to the NW cohort were carried out to identify risk factors for developing at least one post-trauma complication. Complications that were included in the dependent variable of the regression were ventilator-associated pneumonia, unplanned return to the operating room, unplanned admission to intensive-care unit, unplanned intubation, superficial surgical site infection, cerebrovascular accident, severe sepsis, pulmonary embolism, osteomyelitis, organ space surgical site infection, myocardial infarction, extremity compartment syndrome, drug or alcohol withdrawal syndrome, deep vein thrombosis, deep surgical site infection, decubitus ulcer, central line-associated bloodstream infection, catheter-associated urinary tract infection, cardiac arrest with cardiopulmonary resuscitation (CPR), acute respiratory distress syndrome, acute kidney injury, and others. Of note, only significant variables from univariate analyses $(p<0.05)$ were included in our multivariate logistic regression analyses. However, for simplicity, we only showed data for BMI.

\section{Results}

\section{Demographics}

Overall, 41,362 patients from the 2016 ACS TQIP database were collated. Analysis of demographic factors demonstrated that UW patients were the oldest, while MO patients were the youngest $(70.7 \pm 18.3$ years vs. $55.5 \pm 18.9$, Table 1$)$. In this study population, there were more female patients, especially among the UW, NW, and MO cohorts.

\section{Fracture classification and mechanism of injury}

General analysis of femur fracture characteristics showed that the most prevalent injury type was a trochanteric fracture, while the least common injury type was a distal end fracture (33.4\% vs. $14.9 \%$, Table 1). Upon stratification by BMI classifications, head/neck and trochanter fractures were more common among UW patients, while shaft and distal end fractures were more prevalent among obese BMI cohorts (Table 1). More specifically, the rates of head/neck and trochanter femur fractures among UW patients were 39\% and $45 \%$, respectively (Table 1 ). In contrast, the rates of shaft and distal end fractures among UW patients were as low as $10 \%$ and $6 \%$, respectively (Table 1 ).

Additional univariate analysis demonstrated that the MOI varied across all BMI categories. In general, high energy mechanisms of energy, such as MVT, were more prevalent among obese populations, while low energy mechanisms of injury, such as falls, were more prevalent among UW and NW patients. More specifically, falls were responsible for $57 \%$ of all femur fractures among MO patients, while the incidence of falls increased to $79 \%$ in NW patients $(p<0.001$, Table 2$)$ and even $90 \%$ in UW patients (Table 2). In contrast, MVT occupant-related trauma was significantly more prevalent among obese cohorts than NW cohort. For example, the rate of MVT occupant-related trauma was as high as $27.0 \%$ for MO patients and as low as $7 \%$ among NW patients $(p<0.001$, Table 2$)$. Corresponding with our findings, the average ISS was the highest among MO patients (12.5 \pm 6.9 , Table 1), while it was the lowest among UW patients $(10.2 \pm 4.3$, Table 1$)$. Although the difference may not be clinically significant, the vast differences observed in MOI between obese and NW individuals corroborate this finding. 


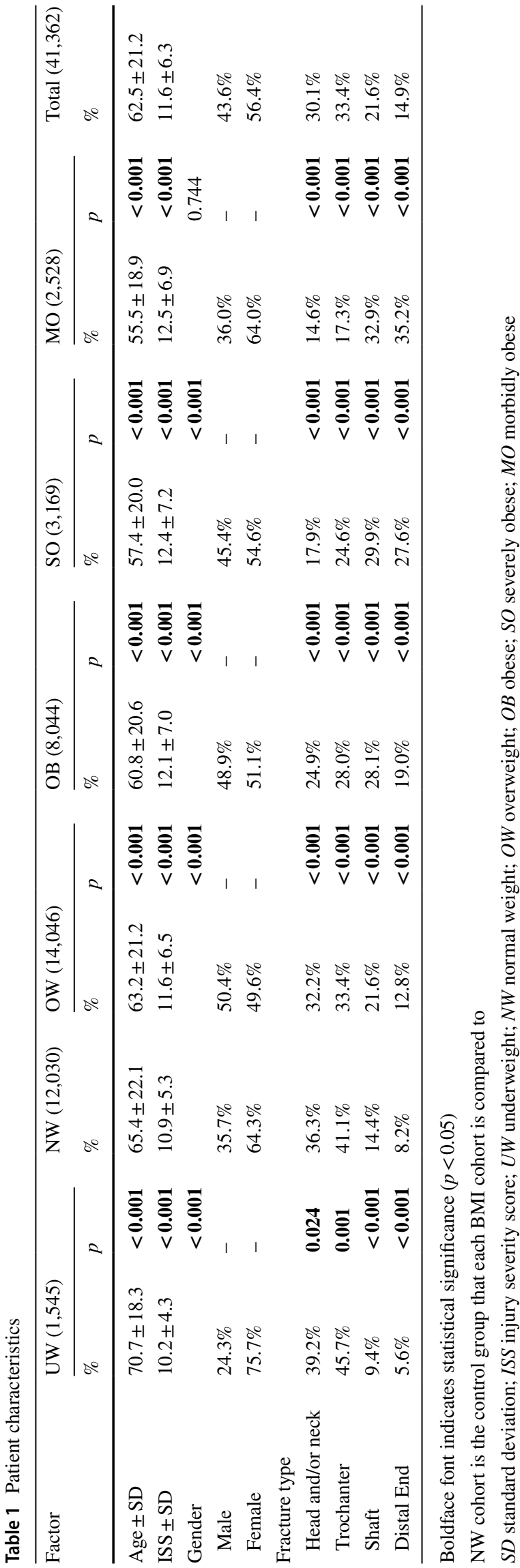

\section{Comorbidities and complications}

Analysis of comorbidities demonstrated that despite being younger than NW patients, obese individuals were associated with higher rates of comorbidities (Table 3). For example, $\mathrm{OB}, \mathrm{MO}$, and SO patients presented with significantly more pre-existing comorbidities, such as diabetes mellitus, chronic renal failure, hypertension, and congestive heart failure (Table 3). Additionally, UW patients presented with significantly more pre-existing comorbidities than NW patients (Table 3). Examples of comorbidities that were more prevalent among UW patients are COPD, disseminated cancer, congestive heart failure, and drug use disorder (Table 3).

After trauma, OB, SO, and MO patients developed significantly more complications, such as acute kidney injury (AKI), acute respiratory distress syndrome (ARDS), deep surgical site infection (SSI), deep vein thrombosis (DVT), and pulmonary embolism (PE) $(p<0.05$, Table 4 and Fig. 1$)$. The rate of developing at least one post-trauma complication was the highest among MO patients, and it was significantly higher than that of NW patients $(20.2 \%$ vs. $14.5 \%, p<0.001$, Table 4). In contrast, there was no difference noted between UW and NW patients for developing at least one post-trauma complication (Table 4). Although, individual complications, such as myocardial infarction, unplanned admission to the ICU, and ventilator-associated pneumonia, were significantly more prevalent among NW patients. Interestingly, UW patients had an admission rate of $1.1 \%$ to the ICU, while this was $1.9 \%$ for NW patients $(1.1 \%$ vs. $1.9 \%, p=0.02$, Table 4).

Multivariate logistic regression of independent factors associated with developing at least one complication demonstrated that, $\mathrm{OB}, \mathrm{SO}$, and MO BMI, as opposed to NW BMI, were associated with a higher risk for developing at least one complication. More specifically, MO patients were associated with about a $45.0 \%$ higher probability of developing a complication $(p<0.001$, Table 5). Additionally, male gender, advanced age and ISS, and various comorbidities were risk factors for developing a complication $(p<0.05$, data not shown).

\section{Discussion}

Among all femur fracture types, patients with $\mathrm{OB}, \mathrm{SO}$, and MO BMI were associated with a higher risk of developing complications in comparison to NW patients. A previous study examining acetabular fractures reported similar observations as patients with higher than NW BMI were more susceptible to developing complications, such as DVT and wound infection [11]. This association was also prevalent among patients with pelvic ring-related injuries $[12,13]$. In contrast, it was unexpected that patients 
Table 2 Mechanism of injury

\begin{tabular}{|c|c|c|c|c|c|c|c|c|c|c|c|}
\hline \multirow[t]{2}{*}{ Factor } & \multicolumn{2}{|c|}{ UW (1545) } & \multirow{2}{*}{$\begin{array}{l}\text { NW }(12,030) \\
\%\end{array}$} & \multicolumn{2}{|c|}{ OW $(14,046)$} & \multicolumn{2}{|c|}{ OB (8044) } & \multicolumn{2}{|c|}{ SO (3169) } & \multicolumn{2}{|c|}{ MO (2528) } \\
\hline & $\%$ & $p$ & & $\%$ & $p$ & $\%$ & $p$ & $\%$ & $p$ & $\%$ & $p$ \\
\hline Fall & 89.6 & $<0.001$ & 79.4 & 70.5 & $<0.001$ & 63.5 & $<0.001$ & 58.7 & $<0.001$ & 57.0 & $<0.001$ \\
\hline Firearm & 1.0 & 0.001 & 2.3 & 3.4 & $<0.001$ & 4.4 & $<0.001$ & 4.8 & $<0.001$ & 2.6 & 0.362 \\
\hline MVT motorcyclist & 0.8 & 0.002 & 2.0 & 4.2 & $<0.001$ & 4.9 & $<0.001$ & 5.9 & $<0.001$ & 3.5 & $<0.001$ \\
\hline MVT occupant & 2.8 & $<0.001$ & 6.6 & 10.8 & $<0.001$ & 16.4 & $<0.001$ & 21.0 & $<0.001$ & 27.0 & $<0.001$ \\
\hline MVT pedal cyclist & 0.2 & 0.863 & 0.2 & 0.1 & 0.067 & 0.1 & 0.058 & 0.0 & 0.105 & 0.0 & 0.07 \\
\hline MVT pedestrian & 1.0 & 0.016 & 1.8 & 2.2 & 0.022 & 2.3 & 0.018 & 1.9 & 0.877 & 2.0 & 0.5 \\
\hline Pedal cyclist, other & 0.5 & 0.003 & 1.4 & 1.1 & 0.074 & 0.6 & $<0.001$ & 0.3 & $<0.001$ & 0.3 & $<0.001$ \\
\hline
\end{tabular}

Boldface font indicates statistical significance $(p<0.05)$

NW cohort is the control group that each BMI cohort is compared to

$M V T$ motor vehicle trauma; $U W$ underweight; $N W$ normal weight; $O W$ overweight; $O B$ obese; $S O$ severely obese; $M O$ morbidly obese

Table 3 Comorbidities

\begin{tabular}{|c|c|c|c|c|c|c|c|c|c|c|c|}
\hline \multirow[t]{2}{*}{ Comorbidities } & \multicolumn{2}{|c|}{ UW (1545) } & \multirow{2}{*}{$\begin{array}{l}\text { NW }(12,030) \\
\%\end{array}$} & \multicolumn{2}{|c|}{ OW $(14,046)$} & \multicolumn{2}{|c|}{ OB (8044) } & \multicolumn{2}{|c|}{ SO (3169) } & \multicolumn{2}{|c|}{ MO (2528) } \\
\hline & $\%$ & $p$ & & $\%$ & $p$ & $\%$ & $p$ & $\%$ & $p$ & $\%$ & $p$ \\
\hline Alcohol use disorder & 5.6 & 0.931 & 5.6 & 4.3 & $<0.001$ & 3.3 & $<0.001$ & 3.6 & $<0.001$ & 2.8 & $<0.001$ \\
\hline Bleeding disorder & 10.4 & 0.909 & 10.5 & 12.6 & $<0.001$ & 12.7 & $<0.001$ & 12.0 & 0.02 & 11.9 & 0.04 \\
\hline Congestive heart failure & 7.5 & 0.02 & 6.0 & 6.4 & 0.206 & 7.2 & $<0.001$ & 8.1 & $<0.001$ & 9.3 & $<0.001$ \\
\hline Current smoker & 21.2 & 0.024 & 18.8 & 15.1 & $<0.001$ & 15.5 & $<0.001$ & 15.1 & $<0.001$ & 15.5 & $<0.001$ \\
\hline Chronic renal failure & 2.3 & 0.467 & 2.6 & 3.4 & 0.001 & 3.5 & 0.001 & 4.2 & $<0.001$ & 3.9 & 0.001 \\
\hline Stroke/CVA & 3.9 & 0.389 & 4.4 & 4.3 & 0.982 & 4.0 & 0.177 & 3.7 & 0.098 & 3.6 & 0.07 \\
\hline Diabetes mellitus & 9.0 & 0.002 & 11.6 & 20.5 & $<0.001$ & 26.1 & $<0.001$ & 31.5 & $<0.001$ & 36.6 & $<0.001$ \\
\hline Disseminated cancer & 1.9 & 0.001 & 1.0 & 1.0 & 0.721 & 0.6 & 0.004 & 0.6 & 0.036 & 0.6 & 0.083 \\
\hline Hypertension & 48.9 & 0.889 & 48.7 & 50.6 & 0.002 & 52.5 & $<0.001$ & 52.3 & $<0.001$ & 54.8 & $<0.001$ \\
\hline COPD & 21.6 & $<0.001$ & 12.6 & 9.2 & $<0.001$ & 10.5 & $<0.001$ & 11.1 & 0.024 & 14.0 & 0.066 \\
\hline Dementia & 19.4 & 0.001 & 16.0 & 9.8 & $<0.001$ & 6.3 & $<0.001$ & 4.2 & $<0.001$ & 2.8 & $<0.001$ \\
\hline Major psychiatric illness & 15.0 & 0.046 & 13.1 & 11.7 & $<0.001$ & 12.3 & 0.092 & 13.6 & 0.447 & 15.6 & 0.001 \\
\hline Drug use disorder & 2.5 & 0.008 & 3.8 & 3.8 & 0.871 & 4.0 & 0.548 & 4.4 & 0.151 & 3.5 & 0.503 \\
\hline ADD/ADHD & 0.6 & 0.443 & 0.5 & 0.4 & 0.193 & 0.5 & 0.728 & 0.5 & $<0.001$ & 0.5 & 0.92 \\
\hline
\end{tabular}

Boldface font indicates statistical significance $(p<0.05)$

NW cohort is the control group that each BMI cohort is compared to

$C V A$ cerebrovascular accident; $C O P D$ chronic obstructive pulmonary disorder; $A D D$ attention deficit disorder; $A D H D$ attention deficit hyperactivity disorder; $U W$ underweight; $N W$ normal weight; $O W$ overweight; $O B$ obese; $S O$ severely obese; $M O$ morbidly obese

with UW BMI were associated with similar and even lower rates of complications than patients with NW BMI. Previous publications have noted that there is a positive association between osteoporosis among UW populations and proximal femur fractures [14], which we even found was more common among this population. Since the UW cohort had higher rates of osteoporosis and was older and frailer than the NW cohort, we expected to observe higher rates of fractures and complications among UW patients. However, our unexpected finding can be attributed to the fact that UW patients had low rates of high energy trauma accidents. Obese patients, on the other hand, were younger, engaged in more high-energy activities, and sustained more shaft fractures. This likely contributed to their high rate of post-trauma complications.

In corroboration with our finding that $\mathrm{OB}, \mathrm{SO}$, and $\mathrm{MO}$ BMI, as opposed to NW BMI, are risk factors for developing at least one complication, literature suggests that high BMI is a risk factor for several negative health outcomes following injury [15-17]. Pierpont et al. reported that high adiposity, which is associated with high BMI, may lead to diminished capillary density and widespread vascular insufficiencies. These health states have also been associated with more inflammation and micro- and macronutrient deficiencies, which can worsen health and negatively affect wound healing [18]. 
Table 4 Patient complications

\begin{tabular}{|c|c|c|c|c|c|c|c|c|c|c|c|}
\hline \multirow[t]{2}{*}{ Complications } & \multicolumn{2}{|c|}{ UW (1545) } & \multirow{2}{*}{$\begin{array}{l}\text { NW }(12,030) \\
\%\end{array}$} & \multicolumn{2}{|c|}{ OW $(14,046)$} & \multicolumn{2}{|c|}{ OB (8044) } & \multicolumn{2}{|c|}{ SO (3169) } & \multicolumn{2}{|c|}{ MO (2528) } \\
\hline & $\%$ & $p$ & & $\%$ & $p$ & $\%$ & $p$ & $\%$ & $p$ & $\%$ & $p$ \\
\hline Acute kidney injury & 0.3 & 0.123 & 0.6 & 1.0 & 0.001 & 1.5 & $<0.001$ & 1.4 & $<0.001$ & 2.2 & $<0.001$ \\
\hline ARDS & 0.0 & 0.074 & 0.3 & 0.4 & 0.021 & 0.6 & $<0.001$ & 0.7 & $<0.001$ & 1.0 & $<0.001$ \\
\hline CAUTI & 0.3 & 0.722 & 0.4 & 0.4 & 0.632 & 0.5 & 0.142 & 0.7 & 0.019 & 0.8 & 0.002 \\
\hline Deep SSI & 0.0 & 0.724 & 0.1 & 0.2 & 0.003 & 0.4 & $<0.001$ & 0.4 & $<0.001$ & 0.7 & $<0.001$ \\
\hline Deep vein thrombosis & 0.3 & 0.076 & 0.6 & 1.1 & $<0.001$ & 1.4 & $<0.001$ & 1.6 & $<0.001$ & 2.3 & $<0.001$ \\
\hline Pulmonary embolism & 0.1 & 0.107 & 0.4 & 0.8 & $<0.001$ & 1.0 & $<0.001$ & 1.7 & $<0.001$ & 1.5 & $<0.001$ \\
\hline Unplanned admission to the ICU & 1.0 & 0.015 & 1.9 & 2.0 & 0.757 & 2.3 & 0.044 & 3.0 & $<0.001$ & 4.2 & $<0.001$ \\
\hline Unplanned return to the $\mathrm{OR}$ & 0.1 & 0.429 & 0.3 & 0.4 & $\mathbf{0 . 0 3 2}$ & 0.6 & $<0.001$ & 0.9 & $<0.001$ & 1.0 & $<0.001$ \\
\hline Cardiac arrest & 0.3 & 0.209 & 0.6 & 0.8 & 0.048 & 1.0 & 0.001 & 1.3 & $<0.001$ & 1.6 & $<0.001$ \\
\hline Decubitus ulcer & 0.3 & 0.195 & 0.5 & 0.5 & 0.996 & 0.7 & 0.089 & 0.8 & $\mathbf{0 . 0 3 2}$ & 1.5 & $<0.001$ \\
\hline Drug/alcohol withdrawal syndrome & 0.7 & 0.68 & 0.6 & 0.4 & 0.006 & 0.4 & 0.011 & 0.3 & 0.022 & 0.2 & 0.018 \\
\hline Extremity compartment syndrome & 0.0 & 0 & 0.1 & 0.1 & 0.085 & 0.2 & $<0.001$ & 0.3 & $<0.001$ & 0.1 & 0.386 \\
\hline Myocardial infarction & 0.0 & 0.005 & 0.5 & 0.5 & 0.738 & 0.4 & 0.149 & 0.7 & 0.26 & 0.5 & 0.92 \\
\hline Organ space SSI & 0.0 & 0 & 0.0 & 0.1 & 0.434 & 0.2 & 0.005 & 0.2 & 0.006 & 0.1 & 0.133 \\
\hline Severe sepsis & 0.2 & 0.34 & 0.3 & 0.4 & 0.393 & 0.4 & 0.286 & 0.8 & 0.001 & 0.8 & 0.001 \\
\hline Stroke/CVA & 0.2 & 0.262 & 0.4 & 0.4 & 0.82 & 0.5 & 0.092 & 0.5 & 0.428 & 0.4 & 0.873 \\
\hline Superficial SSI & 0.0 & 0.139 & 0.1 & 0.2 & 0.211 & 0.3 & 0.056 & 0.3 & 0.016 & 0.5 & 0.001 \\
\hline Unplanned intubation & 0.0 & $<0.001$ & 1.0 & 1.0 & 0.873 & 1.2 & 0.146 & 1.3 & 0.067 & 1.7 & 0.001 \\
\hline Ventilator associated pneumonia & 0.0 & 0.049 & 0.2 & 0.3 & 0.823 & 0.5 & 0.001 & 0.8 & $<0.001$ & 0.6 & 0.002 \\
\hline At least one complication & 14.4 & 0.989 & 14.4 & 15.5 & 0.013 & $\mathbf{1 7 . 6}$ & $<0.001$ & 20.0 & $<0.001$ & 21.2 & $<0.001$ \\
\hline
\end{tabular}

Boldface font indicates statistical significance $(p<0.05)$

NW cohort is the control group that each BMI cohort is compared to

ARDS acute respiratory distress syndrome; CAUTI catheter-associated urinary tract infection; SSI surgical site infection; ICU intensive-care unit; $O R$ operating room; $C L A S B I$ central line-associated bloodstream infection; $C V A$ cerebrovascular accident; $U W$ underweight; $N W$ normal weight; $O W$ overweight; $O B$ obese; $S O$ severely obese; $M O$ morbidly obese

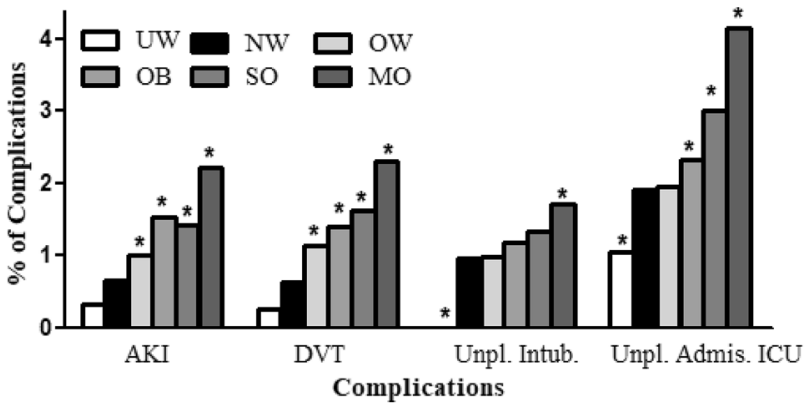

Fig. 1 .A This figure compares the rate of complications for each BMI group to the normal weight BMI group. B (*) denotes statistical significance $(p<0.05)$. C Analyzed complications: acute kidney injury (AKI); deep vein thrombosis (DVT); unplanned intubation (Unpl. Intub.); unplanned admission to intensive care unit (Unpl. Admis. To ICU)

Moreover, MO, SO, and OB patients had higher ISS than NW patients, which is in line with the finding that these patients also sustained injuries from high energy trauma accidents. For example, the rate of MVTs increased with BMI in our univariate analysis. These findings may
Table 5 Multivariate logistic regressions for developing at least one complication

\begin{tabular}{lll}
\hline BMI & OR (CI) & $\mathrm{p}$ \\
\hline Underweight (UW) & $0.983(0.828,1.168)$ & 0.849 \\
Overweight (OW) & $1.024(0.947,1.107)$ & 0.55 \\
Obese (OB) & $1.136(1.039,1.242)$ & $\mathbf{0 . 0 0 5}$ \\
Severely obese (SO) & $1.372(1.221,1.541)$ & $<\mathbf{0 . 0 0 1}$ \\
Morbidly obese (MO) & $1.439(1.263,1.639)$ & $<\mathbf{0 . 0 0 1}$ \\
\hline
\end{tabular}

Six regression analyses are shown, each comparing a BMI subgroup to the NW group

Complication count: UW (233), NW (1,738), OW $(2,184)$, OB (1,414), SO (635), and MO (536)

Boldface font indicates statistical significance $(p<0.05)$

$B M I$ body mass index; $O R$ odds ratio; $C I$ confidence interval

contribute to why obese patients sustained more shaft and distal femur fractures. The shaft and distal femur, along with the knee, especially during motor vehicle trauma, may absorb most of the force from the impact of the dashboard [19]. Since patients with higher BMI were associated more 
high energy traumas, distal and femur fractures, and high ISS, it is comprehensable that these patients developed more complications as a result.

Additionally, male gender, advanced age and ISS, and comorbidities, like congestive heart failure, hypertension, or diabetes mellitus, were also independent risk factors for developing at least one complication. Diabetes mellitus, according to a retrospective review, was associated with a threefold higher rate of complications after ankle fracture surgery [20]. Murray et al. reported that diabetes mellitus may prolong bone healing, decrease metabolism, induce infection, and even decrease cell proliferation [21]. Furthermore, comorbidities in general are associated with being more common among populations with high BMI [22], which partially explains why we observed trends in which complications rates increased as BMI increased in our univariate analysis. Generally, having more comorbidities are indicative of worse overall health status as they may increase one's susceptibility to post-trauma complications [23].

Interestingly, $\mathrm{OB}, \mathrm{SO}$, and $\mathrm{MO}$ higher rates of shaft and distal end fractures. UW on the other hand, sustained more head/neck and trochanter fractures. Previous studies reported that having a high BMI may be advantageous as there is more soft-tissue padding to protect against injury [9]. It is plausible that patients with high BMI were protected from sustaining head/neck and trochanter fractures due to having more subcutaneous tissue that can absorb more force, especially during motor vehicle accidents. Femoral shaft and distal fractures on the other hand, are one of the most common and severe fracture types [24]. These fractures are likely sustained from high energy mechanisms of injury [24], which may pre-dispose patients to developing more complications. Since patients with higher than NW BMI were associated with sustaining more shaft and distal end fractures from high energy traumas, these factors likely contributed to their high complication rate.

This study does have some limitations. Since the design is retrospective, our data analysis was dependent on strict data points collected a national database. As a result, any missing or negative values had to be excluded from our multivariate logistic regression.

\section{Conclusion}

Irrespective of presenting with more comorbidities and sustaining high energy injuries, $\mathrm{OB}, \mathrm{SO}$, and $\mathrm{MO}$ patients, as opposed to NW patients, were associated with having a higher risk of developing complications following a femoral fracture. Overall, better clinical outcomes are observed among patients with no underlying conditions and NW BMI.

Funding This research did not receive funding.
Data availability The data were from the ACS TQIP database.

\section{Declarations}

Conflict of interest The authors of this study have no conflicts of interest.

Ethical approval We received approval from our Institutional Review Board.

\section{References}

1. Chang A, Breeland G, Hubbard J. Anatomy, bony pelvis and lower limb. femur. Treasure Island: StatPearls; 2020.

2. Court-Brown CM, Caesar B. Epidemiology of adult fractures: a review. Injury. 2006;37(8):691-7.

3. Lane NE. Epidemiology, etiology, and diagnosis of osteoporosis. Am J Obstet Gynecol. 2006;194(2 Suppl):S3-11.

4. Vestergaard P, Rejnmark L, Mosekilde L. Hypertension is a risk factor for fractures. Calcif Tissue Int. 2009;84(2):103-11.

5. Vestergaard P, Rejnmark L, Mosekilde L. Relative fracture risk in patients with diabetes mellitus, and the impact of insulin and oral antidiabetic medication on relative fracture risk. Diabetologia. 2005;48(7):1292-9.

6. Body JJ. Increased fracture rate in women with breast cancer: a review of the hidden risk. BMC Cancer. 2011;11:384.

7. Dhungel V, Liao J, Raut H, Lilienthal MA, Garcia LJ, Born J, et al. Obesity delays functional recovery in trauma patients. J Surg Res. 2015;193(1):415-20.

8. Newell MA, Bard MR, Goettler CE, Toschlog EA, Schenarts PJ, Sagraves SG, et al. Body mass index and outcomes in critically injured blunt trauma patients: weighing the impact. J Am Coll Surg. 2007;204(5):1056-61 (discussion 62-4).

9. Tang X, Liu G, Kang J, Hou Y, Jiang F, Yuan W, et al. Obesity and risk of hip fracture in adults: a meta-analysis of prospective cohort studies. PLoS ONE. 2013;8(4): e55077.

10. Weir C, Jan A. BMI classification percentile and cut off points. Treasure Island: StatPearls; 2020.

11. Karunakar MA, Shah SN, Jerabek S. Body mass index as a predictor of complications after operative treatment of acetabular fractures. J Bone Jt Surg Am. 2005;87(7):1498-502.

12. Morris BJ, Richards JE, Guillamondegui OD, Sweeney KR, Mir HR, Obremskey WT, et al. Obesity increases early complications after high-energy pelvic and acetabular fractures. Orthopedics. 2015;38(10): $8881-7$.

13. Sems SA, Johnson M, Cole PA, Byrd CT, Templeman DC, Group MOT. Elevated body mass index increases early complications of surgical treatment of pelvic ring injuries. J Orthop Trauma. 2010;24(5):309-14.

14. Court-Brown CM, Duckworth AD, Ralston S, McQueen MM. The relationship between obesity and fractures. Injury. 2019;50(8):1423-8.

15. Bryant MK, Parrish M, Roy S, Udekwu P, Farrell M, Schinco M, et al. Inferior clinical outcomes after femur fracture in the obese are potentially preventable. Injury. 2019;50(11):2049-54.

16. Kempegowda H, Richard R, Borade A, Tawari A, Graham J, Suk $\mathrm{M}$, et al. Obesity is associated with high perioperative complications among surgically treated intertrochanteric fracture of the femur. J Orthop Trauma. 2017;31(7):352-7.

17. Weinlein JC, Deaderick S, Murphy RF. Morbid obesity increases the risk for systemic complications in patients with femoral shaft fractures. J Orthop Trauma. 2015;29(3):e91-5. 
18. Pierpont YN, Dinh TP, Salas RE, Johnson EL, Wright TG, Robson $\mathrm{MC}$, et al. Obesity and surgical wound healing: a current review. ISRN Obes. 2014;2014:638936.

19. Fadl SA, Sandstrom CK. Pattern recognition: a mechanism-based approach to injury detection after motor vehicle collisions. Radiographics. 2019;39(3):857-76.

20. Blotter RH, Connolly E, Wasan A, Chapman MW. Acute complications in the operative treatment of isolated ankle fractures in patients with diabetes mellitus. Foot Ankle Int. 1999;20(11):687-94.

21. Murray CE, Coleman CM. Impact of diabetes mellitus on bone health. Int J Mol Sci. 2019;20(19):4873.
22. Pantalone KM, Hobbs TM, Chagin KM, Kong SX, Wells BJ, Kattan MW, et al. Prevalence and recognition of obesity and its associated comorbidities: cross-sectional analysis of electronic health record data from a large US integrated health system. BMJ Open. 2017;7(11): e017583.

23. Kirshenbom D, Ben-Zaken Z, Albilya N, Niyibizi E, Bala M. Older age, comorbid illnesses, and injury severity affect immediate outcome in elderly trauma patients. J Emerg Trauma Shock. 2017;10(3): 146-50.

24. Denisiuk M, Afsari A. Femoral shaft fractures. Treasure Island: StatPearls; 2020. 